\title{
Collapse Postulate for Observables with Continuous Spectra
}

\author{
M. D. Srinivas ${ }^{\star}$ \\ International Centre for Theoretical Physics, Trieste, Italy, and Department of Theoretical Physics, \\ University of Madras, Guindy Campus, Madras 600 025, India
}

\begin{abstract}
In order to provide a mathematical framework for discussing the statistical correlations between the outcomes, when an arbitrary sequence of observables are measured, it is necessary to generalize the conventional von Neumann-Lüders collapse postulate to observables with a continuous spectrum. It is shown that the standard prescription in conventional quantum theory for the joint probabilities of compatible observables is sufficient to characterize, more or less completely, the appropriate "generalized collapse postulate" which associates with each observable a unique "finitely additive expectation valued measure". An interesting feature of the collapse associated with observables with continuous spectra, which again follows from the basic principles of conventional quantum theory, is that it must be formulated in terms of the so-called non-normal conditional expectations, which implies that the joint probabilities associated with successive observations of such observables are not in general $\sigma$-additive. The implications of this non- $\sigma$ additivity on the determination of expectation values, correlation functions etc., are also investigated. It is demonstrated that the basic prescriptions introduced in this paper constitute a natural completion of the framework of conventional quantum theory for discussing the statistics of an arbitrary sequence of observations.
\end{abstract}

\section{Introduction}

One of the major problems of the quantum theory of measurement, which has eluded a satisfactory solution so far, has been that of extending the collapse postulate to observables with a continuous spectrum. It is well known that the collapse postulate plays a very crucial role in quantum theory in any discussion of the statistics of the outcomes of a sequence of observations performed on a system. However, the collapse postulate as introduced by von Neumann [1] and later

* Partially supported by University Grants Commission, India 
modified by Lüders [2-7] is appropriate only for observables with a purely discrete spectrum. The main difficulty with observables with continuous spectra appears to be the fact that there does not exist any (atomic) spectral projector corresponding to a single spectral value in the continuum. It is in fact this feature which forces us to modify or generalize the von Neumann-Lüders (or the conventional) collapse postulate so that it can be applied for any arbitrary observable. Stated this way, the basic problem is of course of a purely technical nature. However, it is hardly possible to exaggerate the physical significance of generalizing the collapse postulate for observables with continuous spectra. Most of the observables one deals with ordinarily in either non-relativistic quantum mechanics or in quantum field theory are those with a continuous spectrum and the absence of an appropriate generalization of the collapse postulate has left the theory in a very incomplete state. For example, one cannot employ the conventional collapse postulate to discuss, say, the joint probability distribution associated with two (successive) position measurements carried out on a particle at different times. Another important example is provided by the fact that it has not been possible to systematically discuss from basic principles much of the traditional wisdom that is associated with the Heisenberg uncertainty principle (see for example $[6,8]$ ), for that would necessarily involve a discussion of the measurement of the momentum of a particle (immediately) following the measurement of its position and vice versa. In fact it may be said that the absence of a general collapse postulate constitutes a very serious limitation of the conventional formulation of quantum theory, particularly in connection with the description of successive observations.

In the present investigation we shall arrive at an appropriate generalization of the collapse postulate which of course reduces to the conventional (von Neumann-Lüders) collapse postulate in the case of observables with a purely discrete spectrum. In Sect. II we discuss the reasons why some of the attempts to obtain a direct or immediate generalization of the conventional collapse postulate have not succeeded. It is thus seen that one must attempt at a rather non-trivial generalization after carefully studying the essential mathematical features of the conventional postulate. In fact, it was on the basis of such a study that Davies and Lewis $[9,10]$ suggested that the collapse associated with each observable may be formulated in terms of the so-called operation-valued measures. However, in their investigation the problem of fixing a particular rule of association between the observables and the operation-valued measures (which is the essential content of a collapse postulate) has been left very much open.

In Sect. III we outline a generalization of the conventional framework of quantum theory wherein it is assumed that the collapse associated with each observable may be formulated in terms of a certain "finitely additive expectationvalued measure". This framework includes the proposal of Davies and Lewis as a a particular case. It is shown in Sect. IV that the basic prescription of conventional quantum theory for the joint probabilities of compatible observables $[1,11]$ (a prescription which shall be referred to as the "generalized Born statistical formula (GBSF)"), precludes the possibility of formulating the collapse postulate in terms of operation-valued measures. However, it is seen that the GBSF also serves to characterize, more or less completely, an appropriate generalization of 
the collapse postulate (in terms of the so-called non-normal conditional expectations) which is introduced in Sect. V. Another important consequence of the GBSF is that the joint probabilities of (a successive observation of) observables with continuous spectra, do not in general satisfy the property of $\sigma$-additivity. Sect. VI contains a brief discussion of some of the important consequences of this non- $\sigma$-additivity of probabilities for the statistics of successive observations in quantum theory.

It should be noted that there have also been alternative attempts at generalizing the von Neumann-Lüders collapse postulate by introducing certain "generalized eigenvectors" corresponding to spectral values in the continuum. It has often been suggested [12-18] that one may employ a rigged Hilbert space model (or a related framework) for quantum theory, wherein it can be shown that there does exist an entire set of such generalized eigenvectors, provided we choose an appropriate class of self-adjoint operators as the observables of the theory. Apart from the fact that one must arbitrarily restrict the class of observables, our main objection to the above approach is that it has not led to any version of the collapse postulate which would enable us to predict the joint probabilities of successive observations. This, however, is not the case with a recent proposal due to Farrukh [19], wherein a non-standard extension of the Hilbert space has been employed for generalizing the collapse postulate. Though Farrukh has employed his generalized collapse postulate to calculate the joint probabilities for some interesting particular cases, no general prescription emerges from his work for either the joint probabilities or the expectation values and correlation functions when an arbitrary sequence of observables is being measured. We hope to show that our approach, on the contrary, leads to a more or less natural extension of the von NeumannLüders collapse postulate, from which there also emerges a completely general prescription for the joint probabilities as well as the entire statistics of successive observations in quantum theory.

\section{The Conventional Collapse Postulate and the Difficulties with Continuous Spectrum}

In the conventional formulation of quantum theory [1] the states of a system are characterized by density operators (positive trace class operators of unit trace) on a separable Hilbert space $\mathscr{H}$, and the observables are characterized by selfadjoint operators on $\mathscr{H}$. Then the basic empirical prescription of the theory is given by the following "Born statistical formula (BSF)" for the probability $\operatorname{Pr}_{A}^{\rho}(\Delta)$ that the outcome of an experiment to observe $A$ on an ensemble of systems in state $\rho$ lies in the Borel set $\Delta \in B(R)$ :

$$
\operatorname{Pr}_{A}^{\rho}(\Delta)=\operatorname{Tr}\left(\rho P^{A}(\Delta)\right)
$$

where $\Delta \rightarrow P^{A}(\Delta)$ is the unique spectral measure associated with the self-adjoint operator $A$. It should be borne in mind that in non-relativistic quantum mechanics each observable (in the Heisenberg picture) is associated with an instantaneous observation. Thus the BSF (2.1) gives a prescription for the probabilities associated with a single instantaneous observation only. 
In order to discuss the statistical correlations between the outcomes of a sequence of observations, it is essential to introduce the collapse postulate which (in the conventional framework) "fixes" the state of an ensemble after an observation has been carried out. The generally accepted version of the von NeumannLüders collapse postulate may be stated as follows.

If $\rho$ is the state of an ensemble immediately prior to a measurement of an observable $A$, with the spectral resolution

$$
A=\sum_{i} \lambda_{i} P_{i}
$$

then the state, immediately after measurement, of the (sub) ensemble of all those systems for which the outcome of the measurement was found to lie in the Borel set $\Delta \in B(R)$, is given by the density operator $\mathscr{2}^{A}(\Delta) \rho / \operatorname{Tr}\left[\mathscr{Q}^{A}(\Delta) \rho\right]$, where

$$
\mathscr{2}^{A}(\Delta) \rho=\sum_{\lambda_{i} \in \Delta} P_{i} \rho P_{i} .
$$

The BSF can now be used in conjunction with the above collapse postulate to yield the joint probabilities $\operatorname{Pr}_{A_{1}\left(t_{1}\right), A_{2}\left(t_{2}\right), \ldots, A_{r}\left(t_{r}\right)}^{\rho}\left(\Delta_{1}, \Delta_{2}, \ldots, \Delta_{r}\right)$ that the values of the observables $\left\{A_{i}\left(t_{i}\right)\right\}$ are observed to lie in the Borel sets $\left\{\Delta_{i}\right\}$ when an ensemble of systems originally prepared in state $\rho$ is subjected to the sequence of observations $\left\{A_{1}\left(t_{1}\right), A_{2}\left(t_{2}\right), \ldots, A_{r}\left(t_{r}\right)\right\}$ provided each of the observables $\left\{A_{i}\left(t_{i}\right)\right\}$ has a purely discrete spectrum. If each of the observables $\left\{A_{i}\left(t_{i}\right)\right\}$ has the spectral resolution

$$
A_{i}\left(t_{i}\right)=\sum_{j} \dot{\lambda}_{j}^{(i)} P_{j}^{(i)}\left(t_{i}\right)
$$

and $t_{1}<t_{2}<\ldots<t_{r}$, then we have the following formula due to Wigner [20-22]:

$$
\operatorname{Pr}_{A_{1}\left(t_{1}\right), A_{2}\left(t_{2}\right), \ldots, A_{r}\left(t_{r}\right)}^{\rho}\left(\Delta_{1}, \Delta_{2}, \ldots, \Delta_{r}\right)=\operatorname{Tr}\left[\mathscr{2}^{A_{r}\left(t_{r}\right)}\left(\Delta_{r}\right) \ldots \mathscr{2}^{A_{2}\left(t_{2}\right)}\left(\Delta_{2}\right) \mathscr{Q}^{A_{1}\left(t_{1}\right)}\left(\Delta_{1}\right) \rho\right],
$$

where

$$
\mathscr{Q}^{A_{i}\left(t_{i}\right)}\left(\Delta_{i}\right)=\sum_{\lambda_{j}^{(i)} \in \Delta_{i}} P_{j}^{(i)}\left(t_{i}\right) \rho P_{j}^{(i)}\left(t_{i}\right),
$$

for each $i \in\{1,2, \ldots, r\}$.

It has also been noted by Wigner $[22,23]$ that Eq. (2.5) (to be referred to as the Wigner formula) combines in itself all the observational content of both the BSF and the collapse postulate as long as we restrict ourselves to observables with purely discrete spectra. In fact, for such observables, the Wigner formula (2.5) may itself be adopted as the fundamental statistical prescription of the theory-so that there is no longer any need for considering the collapse postulate or the BSF separately. It may also be noted that the joint probabilities (2.5) exhibit a certain "quantum interference of probabilities" [24-26], which also demonstrates that they cannot be brought under the purview of classical probability theory.

The major difficulty in formulating a completely general (quantum) theory of successive observations is that the collapse postulate as stated above does not 
make any sense for an observable with a continuous spectrum. Since there are no eigenvectors (in the Hilbert space) associated with spectral values in the continuum, Eq. (2.3) does not make any sense for observables with continuous spectra as the projectors $P_{i}$ do not exist. Therefore the major problem appears to be a purely technical one of extending the sum in Eq. (2.3) to some sort of an integral which would make sense for any arbitrary spectral measure on the real line. We have already emphasised the physical significance of the above problem, which also suggests that an appropriate solution should be based on the fundamental principles that underlie quantum theory.

Before going into a general analysis of the problem, we would like to note that there have been some misconceptions concerning this problem in the literature, which have not been subjected to a proper critical examination. For example, one suggestion which is implicit even in some textbooks is to replace the transformations $\left\{\mathscr{Q}^{A}(\Delta)\right\}$ of Eq. (2.3) by the transformations $\left\{\overline{2}^{A}(\Delta)\right\}$ as given below

$$
\overline{\mathscr{2}}^{A}(\Delta)=P^{A}(\Delta) \rho P^{A}(\Delta)
$$

where $\Delta \rightarrow P^{A}(\Delta)$ is the spectral measure associated with the observable $A \cdot{ }^{1}$ Unlike (2.3), Eq. (2.7) is of course meaningful for all observables, and it is also consistent with the BSF, as we have

$$
\operatorname{Pr}_{A}^{\rho}(\Delta)=\operatorname{Tr}\left[\overline{\mathscr{Q}}^{A}(\Delta) \rho\right]=\operatorname{Tr}\left[P^{A}(\Delta) \rho P^{A}(\Delta)\right]=\operatorname{Tr}\left(\rho P^{A}(\Delta)\right),
$$

in agreement with (2.1). However, the unphysical character of the above proposal shows up very clearly once we employ it to evaluate the joint probabilities of successive observations. We are led in the same way as before to formula (2.5) with the only difference that $\mathscr{2}^{A_{2}\left(t_{i}\right)}\left(\Delta_{i}\right)$ are now to be replaced by $\overline{\mathscr{Q}}^{A_{i}\left(t_{i}\right)}\left(\Delta_{i}\right)$. However, as the $\overline{2}^{A_{l}\left(t_{i}\right)}\left(\Delta_{i}\right)$ are not finitely additive in $\Delta_{i}$, it can easily be shown that the joint probabilities turn out to be not even finitely additive. For example, if $A, B$ are two incompatible observables (i.e. the associated spectral projectors do not commute with each other) and $\Delta, \Delta_{1}, \Delta_{2} \in B(R)$ are such that $\Delta_{1} \cap \Delta_{2}=\varnothing$, then Eq. (2.7) leads to

$$
\begin{aligned}
\operatorname{Pr}_{A, B}^{\rho}\left(\Delta_{1} \cup \Delta_{2}, \Delta\right) & =\operatorname{Tr}\left[\overline{\mathscr{Q}}^{B}(\Delta) \overline{\mathscr{Q}}^{A}\left(\Delta_{1} \cup \Delta_{2}\right) \rho\right] \\
& =\operatorname{Tr}\left(P^{B}(\Delta) P^{A}\left(\Delta_{1} \cup \Delta_{2}\right) \rho P^{A}\left(\Delta_{1} \cup \Delta_{2}\right)\right),
\end{aligned}
$$

which is in general different from

$$
\begin{aligned}
\operatorname{Pr}_{A, B}^{\rho}\left(\Delta_{1}, \Delta\right)+\operatorname{Pr}_{A, B}^{\rho}\left(\Delta_{2}, \Delta\right)= & \operatorname{Tr}\left[\overline{\mathscr{Q}}^{B}(\Delta) \overline{\mathscr{Q}}^{A}\left(\Delta_{1}\right) \rho\right]+\operatorname{Tr}\left[\overline{\mathscr{Q}}^{B}(\Delta) \overline{\mathscr{Q}}^{A}\left(\Delta_{2}\right) \rho\right] \\
= & \operatorname{Tr}\left[P^{B}(\Delta) P^{A}\left(\Delta_{1}\right) \rho P^{A}\left(\Delta_{1}\right)\right] \\
& +\operatorname{Tr}\left[P^{B}(\Delta) P^{A}\left(\Delta_{2}\right) \rho P^{A}\left(\Delta_{2}\right)\right] .
\end{aligned}
$$

This lack of finite additivity of the joint probabilities makes the above proposal of replacing Eq. (2.3) by Eq. (2.7) completely untenable.

In his classic treatise [1] on quantum mechanics, von Neumann considered

1 Such a version of the collapse postulate is implicit for example in all those treatments of localization (or position measurement), where the wave function $\psi(x)$ of a particle is assumed to be transformed into $\chi_{\Delta}(x) \psi(x)$ modulo the normalization factor (where $\chi_{\Delta}(x)$ is the characteristic function of $\Delta \in B(R)$ ), the moment the particle is found to be localized in $\Delta$. 
another way of generalizing the collapse postulate to observables with continuous spectra. He suggested that we may consider functions of these observables which have a purely discrete spectrum and employ Eq. (2.3) for the collapse associated with such functions as some sort of an approximation to the collapse associated with the original observable. It must be stated that this programme has not been actually carried out even for particular cases, and therefore we do not have any mathematically precise characterization of the nature of the approximation or its consistency. However, apart from the fact that von Neumann himself considered this as just a tentative solution (cf. the footnote on p. 223 of [1]), there are very strong reasons to suspect that such a programme may not be viable. The procedure advocated by von Neumann involves partitioning the spectrum of an observable into a countable collection of disjoint intervals. For example, if $A$ is an observable with a continuous spectrum we may consider the observable $f_{\alpha}(A)$, where for each $\alpha>0$

$$
f_{\alpha}(x)=\sum_{n=-\infty}^{\infty}\left(n+\frac{1}{2}\right) \alpha \chi_{[n \alpha,(n+1) \alpha)}(x),
$$

where $\chi_{\mathrm{I}},(x)$ denotes the characteristic function of the interval [ $)$. The observable $f_{\alpha}(A)$ has a purely discrete spectrum and a precise measurement of $f_{\alpha}(A)$ is supposed to be equivalent to an approximate measurement of $A$ with an apparatus which has a limit of resolution given by $\alpha$.

However, the crucial point is that there is hardly any justification for the claim that the collapse associated with $f_{\alpha}(A)$ is in some sense related to that associated with $A$, however small the resolution $\alpha$ might be. Of course, the spectral projectors of $A$ and its function $f(A)$, where $f: R \rightarrow R$ is a Borel function, are related by the equation

$$
P^{f(A)}(\Delta)=P^{A}\left(f^{-1}(\Delta)\right),
$$

for all $\Delta \in B(R)$. However, Eq. (2.9) also shows that in general the spectral projectors of $f(A)$ do not determine all the spectral projectors of $A$. As regards the collapse associated with $A$ and $f(A)$ the situation is very similar, at least as regards observables with purely discrete spectra. In fact, we have the following lemma which is a direct consequence of Eqs. (2.2) and (2.3).

Lemma 2.1. If $A$ is an observable with a purely discrete spectrum, then $\left\{\mathscr{Q}^{f(A)}(\Delta)\right\}$ and $\left\{2^{A}\left(f^{-1}(\Delta)\right)\right\}$ are totally unrelated, unless $f: R \rightarrow R$ is a Borel function which is injective on the spectrum of $A$, in which case we have

$$
2^{f(A)}(\Delta)=\mathscr{Q}^{A}\left(f^{-1}(\Delta)\right)
$$

for all $\Delta \in B(R)$.

The requirement that $f: R \rightarrow R$ be injective on the spectrum of $A$ is essentially that we have

$$
f(x)=f(y) \Rightarrow x=y
$$

for $x, y$ lying in the spectrum of $A$.

From the above lemma we can easily see that the collapse associated with 
$f_{\alpha}(A)$ is very different from even that associated with, say, $f_{2 \alpha}(A)$. In other words, even a change in the so-called resolution of the apparatus gives rise to a completely unrelated collapse expression. Also, as Davies and Lewis [9] have noted, any approach based on a partitioning of the spectrum into disjoint intervals is rather suspect, as it often destroys the group covariance properties possessed by the observable. Finally, the result of Lemma 2.1 suggests that in the general case also we have every reason to suspect that the collapse associated with $A$ and $f(A)$ are unrelated, unless the function $f$ is injective on the spectrum of $A$. Therefore we cannot hope to get any idea about the collapse associated with an observable $A$ with a continuous spectrum by studying the collapse associated with functions of the form $f_{\alpha}(A)$, for $f_{\alpha}$ takes constant values on each of the intervals $[n \alpha,(n+1) \alpha)$.

From the preceding discussion it becomes clear that the appropriate generalization of the conventional collapse postulate to observables with continuous spectra should involve a completely non-trivial generalization of Eq. (2.3). Several other arguments in favour of this point have also been provided by Davies [27]. It would be appropriate here to recall his conclusion $([27]$, p. 58$)$ that "... in measurement theory discrete and continuous projection-valued measures have very different properties. This runs completely counter to the frequent suggestion that for foundational purposes one need only consider discrete observables, the continuous ones being approximated by discrete ones in some manner".

\section{The Basic Framework for Generalizing the Collapse Postulate}

In order to gain an insight into the problem of generalizing the conventional collapse postulate, it is very necessary to first analyse its essential mathematical features as contained in Eqs. (2.2) and (2.3). It was noted by Schwinger [28] that the collapse or measurement transformation $\rho \rightarrow \mathscr{2}^{A}(\Delta) \rho$ as given by $(2.3)$ can be viewed as a positive linear transformation on the space of trace class operators. Later, Haag and Kastler [29] introduced the notion of an "operation" which incorporates all the essential mathematical characteristics of such collapse transformations. In order to define the notion of an operation, let us consider the Banach space $\mathscr{T}(\mathscr{H})$ (under the trace norm) of all trace class operators on $\mathscr{H}$. The set $\mathscr{T}^{+}(\mathscr{H})$ of all non-negative trace class operators is a norm-closed cone in $\mathscr{T}(\mathscr{H})$. An operation $\mathscr{Q}$ is a linear self-adjoint positive map $\mathscr{Q}: \mathscr{T}(\mathscr{H}) \rightarrow \mathscr{T}(\mathscr{H})$, which is also norm-non-increasing, in that

$$
\operatorname{Tr}(\mathscr{Q}(v)) \leqq \operatorname{Tr}(v)
$$

for all $v \in \mathscr{T}^{+}(\mathscr{H})$. It is easy to see that the transformations $\rho \rightarrow \mathscr{Q}^{A}(\Delta) \rho$ as defined in Eq. (2.3) can be uniquely extended into operations. We shall denote the set of all operations associated with $\mathscr{H}$ as $\mathscr{Q}(\mathscr{H})$.

It was noted by Davies and Lewis [9] that the statement of the conventional collapse postulate essentially associates an operation-valued measure with each observable. An operation-valued measure (on the real line) is an association $\Delta \rightarrow \mathscr{Q}(\Delta)$ of $B(R)$ into $\mathscr{Q}(\mathscr{H})$ such that the following properties are satisfied:

i) $\operatorname{Tr}(\mathscr{Q}(R) v)=\operatorname{Tr}(v)$, for all $v \in \mathscr{T}^{+}(\mathscr{H})$; 
ii) If $\left\{\Delta_{i}\right\}$ is a sequence of mutually disjoint elements of $B(R)$, then

$$
\mathscr{2}\left(\bigcup_{i} \Delta_{i}\right)=\sum_{i} \mathscr{2}\left(\Delta_{i}\right)
$$

where the sum on the right-hand side converges in the strong operator topology on $\mathscr{Q}(\mathscr{H})$.

It is rather straightforward to verify that for an observable $A$ with a purely discrete spectrum (as given by (2.2)), the association $\Delta \rightarrow \mathscr{Q}^{A}(\Delta)$ (as given by (2.3)) defines an operation-valued measure. Thus the conventional collapse postulate may now be viewed as a statement associating an operation-valued measure $2^{A}: B(R) \rightarrow \mathscr{Q}(\mathscr{H})$, with each observable $A$ with a purely discrete spectrum. The first important step in generalizing the above postulate to arbitrary observables appears to be the suggestion of Davies and Lewis [9] that the collapse associated with every observable could be formulated in terms of operation-valued measures. They also pointed out that associated with each self-adjoint operator $A$, there are in fact several operation-valued measures $\mathscr{Q}^{A}: B(R) \rightarrow \mathscr{2}(\mathscr{H})$, each of which satisfies the requirement imposed by BSF that

$$
\operatorname{Tr}\left(\mathscr{Q}^{A}(\Delta) \rho\right)=\operatorname{Pr}_{A}^{\rho}(\Delta)=\operatorname{Tr}\left(\rho P^{A}(\Delta)\right)
$$

for each density operator $\rho$. Also, once a particular association $A \rightarrow 2^{A}$ between the observables and the operation-valued measures is specified, then the Wigner formula (2.5) can be employed to obtain the joint probabilities of successive observations.

However, we are still left with the problem of fixing the association $A \rightarrow 2^{A}$ between the observables and the operation-valued measures. As we noted already, the BSF does not by itself characterize the above association uniquely. We are therefore led back to a careful examination of the basic principles of conventional quantum theory in the hope that further light may be thrown on the problem of obtaining the appropriate generalization of the collapse postulate. Since the generalized collapse postulate is essential only insofar as it makes possible a discussion of the statistical correlations between the outcomes of successive observations, our goal shall be to obtain an appropriate generalization of the Wigner formula (2.5). We shall first recast the Wigner formula in an alternative form, which will enable us to consider a more general class of mathematical objects (for the generalization of the collapse postulate) than is provided by the class of operation-valued measures. As we shall see later, this constitutes a very essential step in the generalization of the collapse postulate.

We employ the well-known duality between the Banach space $\mathscr{T}(\mathscr{H})$ and its dual $B(\mathscr{H})$ (the Banach space under operator norm of all bounded operators on $\mathscr{H}$ ), which can be expressed in terms of the bilinear form

$$
\langle v, A\rangle=\operatorname{Tr}(v A),
$$

for $v \in \mathscr{T}(\mathscr{H})$ and $A \in B(\mathscr{H})$. The cone of non-negative operators in $B(\mathscr{H})$ will be denoted as $B^{+}(\mathscr{H})$. We define an "expectation" to be a map $\mathscr{E}: B(\mathscr{H}) \rightarrow B(\mathscr{H})$ which is linear, self-adjoint positive and satisfies

$$
0 \leqq \mathscr{E}(I) \leqq I
$$


where $I$ is the identity operator on $\mathscr{H}$. We should note that the term "expectation" is also used for several different notions in the literature. The set of all expectations associated with $\mathscr{H}$ will be denoted as $\mathscr{E}(\mathscr{H})$.

An expectation $\mathscr{E}$ is said to be normal if it is also continuous under the ultraweak topology on $B(\mathscr{H})$. Let $\mathscr{E}_{\sigma}(\mathscr{H})$ denote the set of all normal expectations. It is well known [27] that with each operation $\mathscr{2} \in \mathscr{Q}(\mathscr{H})$, there is associated a unique adjoint $\mathscr{2}^{*} \in \mathscr{E}_{\sigma}(\mathscr{H})$ (and vice versa) such that

$$
\operatorname{Tr}(\mathscr{Q}(v) A)=\operatorname{Tr}\left(v \mathscr{2}^{*}(A)\right)
$$

for all $v \in \mathscr{T}(\mathscr{H})$ and $A \in B(\mathscr{H})$. We can now recast the Wigner formula (2.5) as follows:

$$
\operatorname{Pr}_{A_{1}\left(t_{1}\right), \ldots, A_{r}\left(t_{r}\right)}^{\rho}\left(\Delta_{1}, \Delta_{2}, \ldots, \Delta_{r}\right)=\operatorname{Tr}\left[\rho\left\{\mathscr{E}^{A_{1}\left(t_{1}\right)}\left(\Delta_{1}\right) \mathscr{E}^{A_{2}\left(t_{2}\right)}\left(\Delta_{2}\right) \ldots \mathscr{E}^{A_{r}\left(t_{r}\right)}\left(\Delta_{r}\right)\right\} I\right],
$$

where

$$
\mathscr{E}^{A_{l}\left(t_{t}\right)}\left(\Delta_{i}\right)=\mathscr{2}^{A_{i}\left(t_{t}\right)}\left(\Delta_{i}^{*}\right),
$$

is the normal expectation which is the adjoint of the operation $\mathscr{2}^{A_{t}\left(t_{t}\right)}\left(\Delta_{i}\right)$ given by Eqs. (2.4) and (2.6). In other words, if the observable $A$ has the spectral resolution

$$
A=\sum_{i} \lambda_{i} P_{i}
$$

then the expectations $\left\{\mathscr{E}^{A}(\Delta)\right\}$ are given by

$$
\mathscr{E}^{A}(\Delta) B=\sum_{\lambda_{i} \in \Delta} P_{i} B P_{i}
$$

for each $\Delta \in B(R), B \in B(\mathscr{H})$. It is again straightforward to verify that the association $\Delta \rightarrow \mathscr{E}^{A}(\Delta)$ as given by Eqs. (3.10) and (3.11) defines a "normal expectation-valued measure" (a notion which was introduced by Davies [10] under the name "expectation"), in that it satisfies the following:

i) For each $\Delta \in B(R), \mathscr{E}^{A}(\Delta) \in \mathscr{E}_{\sigma}(\mathscr{H})$.

ii) $\mathscr{E}^{A}(R) I=\mathrm{I}$.

iii) If $\left\{\Delta_{i}\right\}$ is a sequence of mutually disjoint elements of $B(R)$, then

$$
\mathscr{E}^{A}\left(\bigcup_{i} \Delta_{i}\right) B=\sum_{i} \mathscr{E}^{A}\left(\Delta_{i}\right) B
$$

for all $B \in B(\mathscr{H})$, where the sum on the right-hand side converges in the ultraweak topology on $B(\mathscr{H})$.

It is quite obvious that the normal expectation-valued measures are the duals of the operation-valued measures considered earlier. We shall later find that the basic principles of conventional quantum theory are inconsistent with any formulation of the generalized collapse postulate in terms of the operation-valued measures or, equivalently, in terms of the normal expectation-valued measures. Hence, in our search for the appropriate generalization of the collapse postulate, we shall allow for a more general class of mappings $\Delta \rightarrow \mathscr{E}^{A}(\Delta)$, where the expectations $\left\{\mathscr{E}^{A}(\Delta)\right\}$ (i) are not restricted to be normal, and (ii) are required to be only finitely additive in $\Delta$. The basic framework in which an appropriate generalization 
of the collapse postulated is being sought, can now be stated precisely in terms of the following assumptions $(\mathrm{A})-(\mathrm{C})$ :

A. The states of a system are represented by density operators, and the observables (corresponding to instantaneous measurements) by self-adjoint operators on a separable Hilbert space $\mathscr{H}$.

B. For each observable $A$, there is associated a finitely additive expectationvalued measure $\mathscr{E}^{A}: B(R) \rightarrow \mathscr{E}(\mathscr{H})$, i.e.

1) $\mathscr{E}^{A}(\Delta) \in \mathscr{E}(\mathscr{H})$,

for each $\Delta \in B(R)$.

2) $\mathscr{E}^{A}(R) I=I$.

3) If $\Delta_{1}, \Delta_{2} \in B(R)$ are such that $\Delta_{1} \cap \Delta_{2}=\varnothing$, then

$\mathscr{E}^{A}\left(\Delta_{1} \cup \Delta_{2}\right)=\mathscr{E}^{A}\left(\Delta_{1}\right)+\mathscr{E}^{A}\left(\Delta_{2}\right)$.

C. The joint probability that the outcomes of experiments to measure $\left\{A_{i}\left(t_{i}\right)\right\}$ are found to lie in Borel sets $\left(\Delta_{i}\right)$, when an ensemble of systems prepared in state $\rho$ is subjected to the sequence of experiments $\left\{A_{1}\left(t_{1}\right), A_{2}\left(t_{2}\right), \ldots, A_{r}\left(t_{r}\right)\right\}$, where $t_{1}<t_{2}<\ldots<t_{r}$, is given by the generalized Wigner formula

$$
\operatorname{Pr}_{A_{1}\left(t_{1}\right), A_{2}\left(t_{2}\right), \ldots, A_{r}\left(t_{r}\right)}^{\rho}\left(\Delta_{1}, \Delta_{2}, \ldots, \Delta_{r}\right)=\operatorname{Tr}\left[\rho\left\{\mathscr{E}^{A_{1}\left(t_{1}\right)}\left(\Delta_{1}\right) \mathscr{E}^{\mathscr{A}_{2}\left(t_{2}\right)}\left(\Delta_{2}\right) \ldots \mathscr{E}^{A_{r}\left(t_{r}\right)}\left(\Delta_{r}\right)\right\} I\right] .
$$

A few remarks may be made on the physical motivation behind these assumptions. The assumption (A) needs no comment. In assumption (C) we have adopted as the basic statistical prescription of the theory a generalization of the Wigner formula (2.5), which of course includes as particular cases all those situations which can be dealt with in the framework of conventional quantum theory. The statement of the collapse postulate, in our framework, will be a rule of association $A \rightarrow \mathscr{E}^{A}$ between self-adjoint operators and finitely additive expectation-valued measures. The requirement that for each $\Delta, \mathscr{E}^{A}(\Delta)$ be a linear map is to ensure that the probabilities (3.17) are additive in the density operator $\rho$ under the formation of mixtures. The self-adjointness and positivity of $\mathscr{E} A(\Delta)$ ensure that Eq. (3.17) yields non-negative probabilities. Condition (B2) ensures that the probabilities are normalized to unity and, finally, (B3) ensures that the probabilities are finitely additive. In fact, once the fundamental statistical prescription of the theory is assumed to be in the form given by (3.17), then all the properties of $\left\{\mathscr{E}^{A}(\Delta)\right\}$ which are postulated in assumption $(B)$ can be deduced as necessary consequences of the basic physical requirements on probabilities.

\section{The Generalized Born Statistical Formula and its Incompatibility with $\sigma$-Additive Probabilities}

Having fixed the basic framework of our discussion, we are now in a position to analyse the various constraints imposed on the collapse postulate or the association $A \rightarrow\left\{\mathscr{E}^{A}(\Delta)\right\}$ by the fundamental principles of conventional quantum theory. The first and the foremost requirement is that the generalized Wigner formula (3.17) should yield the same probabilities as the BSF (2.1) for the outcomes 
of a single observation. In other words, we should have

$$
\operatorname{Pr}_{A}^{\rho}(\Delta)=\operatorname{Tr}\left[\rho \mathscr{E}^{A}(\Delta) I\right]=\operatorname{Tr}\left(\rho P^{A}(\Delta)\right)
$$

for all $\Delta \in B(R)$, for all observables $A$ and states $\rho$. It is easy to see that (4.1) is equivalent to the requirement that for each observable $A$,

$$
\mathscr{E}^{A}(\Delta) I=P^{A}(\Delta)
$$

for all $\Delta \in B(R)$. In the literature, the above requirement is usually referred to as the requirement that the observable "determined" by the expectation-valued mapping $\Delta \rightarrow \mathscr{E}^{A}(\Delta)$ should be the projection-valued measure $\Delta \rightarrow P^{A}(\Delta)$. We shall now see that Eq. (4.2) itself imposes rather severe restrictions on the association $A \rightarrow\left\{\mathscr{E}^{A}(\Delta)\right\}$.

For each observable $A$, let us denote by $\mathscr{U}_{A}$ the von Neumann algebra generated by the set $\left\{P^{A}(\Delta) \mid \Delta \in B(R)\right\}$ of the spectral projectors of $A$. It is well known that

$$
\mathscr{U}_{A}=\left\{P^{A}(\Delta) \mid \Delta \in B(R)\right\}^{\prime \prime} .
$$

The commutant $\mathscr{U}_{A}^{\prime}$ of $\mathscr{U}_{A}$ is also given by

$$
\mathscr{U}_{A}^{\prime}=\left\{P^{A}(\Delta) \mid \Delta \in B(R)\right\}^{\prime} .
$$

We now have the following result essentially due to Davies [10], Twareque Ali and Emch [30].

Lemma 4.1. If the BSF (4.2) is also satisfied in addition to the assumptions $(A)-(C)$, then for each observable $A$

$$
\mathscr{E}^{A}(\Delta) B \in \mathscr{U}_{A}^{\prime}
$$

for all $\Delta \in B(R), B \in B(\mathscr{H})$.

Proof. Let $B \in B^{+}(\mathscr{H})$. Since we have

$$
\|B\| I-B \geqq 0,
$$

it follows from (4.2) and (B1) that

$$
0 \leqq \mathscr{E}^{A}(\Delta) \mathrm{B} \leqq\|B\| \mathscr{E}^{A}(\Delta) I=\|B\| P^{A}(\Delta),
$$

for all $\Delta \in B(R)$. From the fact that $\mathscr{E}^{A}(\Delta) B /\|B\|$ is a positive operator majorized by the projector $P^{A}(\Delta)$, it follows that $\mathscr{E} A(\Delta) B$ commutes with $P^{A}(\Delta)$, and we have

$$
\mathscr{E}^{A}(\Delta) B=P^{A}(\Delta) \mathscr{E}^{A}(\Delta)(B)=\mathscr{E}^{A}(\Delta)(B) P^{A}(\Delta) .
$$

Now, if $\Delta_{1}, \Delta_{2} \in B(R)$ and $\Delta_{2}^{\prime}$ is the complement of $\Delta_{2}$ in $R$, then Eq. (4.8) implies the following:

$$
\begin{aligned}
& \mathscr{E}^{A}\left(\Delta_{1} \cap \Delta_{2}\right)(B) P^{A}\left(\Delta_{2}\right)=P^{A}\left(\Delta_{2}\right) \mathscr{E}^{A A}\left(\Delta_{1} \cap \Delta_{2}\right)(B) ; \\
& \mathscr{E}^{A}\left(\Delta_{1} \cap \Delta_{2}^{\prime}\right)(B) P^{A}\left(\Delta_{2}\right)=0 .
\end{aligned}
$$

Adding the above two equations and using the finite additivity (B3) we get

$$
\mathscr{E}^{A}\left(\Delta_{1}\right)(B) P^{A}\left(\Delta_{2}\right)=P^{A}\left(\Delta_{2}\right) \mathscr{E}^{A}\left(\Delta_{1}\right)(B)
$$


for all $\Delta_{1}, \Delta_{2} \in B(R)$ and $B \in B^{+}(\mathscr{H})$, from which the lemma follows immediately.

The other important constraint on the collapse postulate $A \rightarrow\left\{\mathscr{E}^{A}(\Delta)\right\}$ is that for compatible observables, the joint probabilities as predicted by the generalized Wigner formula (3.17) should coincide with those obtained on the basis of the standard prescription for such joint probabilities in conventional quantum theory. It is well known that two bounded observables are said to be compatible if they commute. In the general case (which includes also unbounded observables) two observables $A, B$ are said to be compatible [11] iff

$$
P^{A}\left(\Delta_{1}\right) P^{B}\left(\Delta_{2}\right)=P^{B}\left(\Delta_{2}\right) P^{A}\left(\Delta_{1}\right)
$$

for all $\Delta_{1}, \Delta_{2} \in B(R)$. A set of observables $\left\{A_{1}, A_{2}, \ldots, A_{r}\right\}$ is said to be a compatible set if every pair of elements of the set is compatible. It is well known that apart from the BSF (2.1) and the collapse postulate (2.3), the conventional formulation of quantum theory includes the following generalization $(D)$ of the BSF as one of its basic assumptions $[1,11]$ :

D. Generalized Born statistical formula $(G B S F)$ : If $\left\{A_{1}, A_{2}, \ldots, A_{r}\right\}$ is a compatible set of observables then

$$
\operatorname{Pr}_{A_{1}, A_{2}, \ldots, A_{r}}^{\rho}\left(\Delta_{1}, \Delta_{2}, \ldots, \Delta_{r}\right)=\operatorname{Tr}\left(\rho P^{A_{1}}\left(\Delta_{1}\right) P^{A_{2}}\left(\Delta_{2}\right) \ldots P^{A_{r}}\left(\Delta_{r}\right)\right)
$$

for all $\Delta_{i} \in B(R), i=1,2, \ldots, r$.

It is obvious that the GBSF (4.11) includes the BSF (2.1) as a particular case. In fact GBSF constitutes a non-trivial generalization of the BSF as it happens to be the only available prescription in conventional quantum theory for joint probabilities for observables with a continuous spectrum, and as such plays a very important role in several applications of quantum theory. However, it should be emphasised that Eq. (4.11) holds for compatible observables only, and in any case it cannot be applied otherwise as the right-hand side will then take on complex values. In the following lemma we shall show that the GBSF (4.11) imposes a further constraint on the collapse postulate.

Lemma 4.2. Under the assumptions $(A)-(C)$, the $G B S F(D)$ is equivalent to the requirement that for each observable $A$,

$$
\mathscr{E}^{A}(\Delta) P=P^{A}(\Delta) P
$$

for each $\Delta \in B(R)$ and every projector $P \in \mathscr{U}_{A}^{\prime}$.

Proof. Let $P \in \mathscr{U}_{A}^{\prime}$ be a projector. Now if $\lambda, \mu$ are real numbers, then $B=\lambda P+$ $\mu(I-P)$ is an observable which is compatible with $A$. Then we have from the GBSF (4.11)

$$
\operatorname{Pr}_{A, B}^{\rho}(\Delta,\{\lambda\})=\operatorname{Tr}\left(\rho P^{A}(\Delta) P\right) .
$$

However, from the generalized Wigner formula (3.17) we also have

$$
\operatorname{Pr}_{A, B}^{\rho}(\Delta,\{\lambda\})=\operatorname{Tr}\left(\rho\left\{\mathscr{E}^{A}(\Delta) \mathscr{E}^{B}(\{\lambda\})\right\} I\right)
$$

we have already seen that the BSF, which is a particular case of GBSF, is equivalent to the relation (cf. Eq. (4.2))

$$
\mathscr{E}^{B}(\{\lambda\}) I=P .
$$


We therefore have

$$
\operatorname{Tr}\left(\rho P^{A}(\Delta) P\right)=\operatorname{Tr}\left(\rho \mathscr{E}^{A}(\Delta) P\right)
$$

for all density operators $\rho$, which implies that

$$
\mathscr{E}^{A}(\Delta) P=P^{A}(\Delta) P,
$$

thereby establishing the lemma.

We are now in a position to obtain a more or less complete characterization of the collapse postulate $A-\left\{\mathscr{E}^{A}(\Delta)\right\}$ which follows from the assumptions (A)-(D).

Theorem 4.3. The assumptions $(A)-(D)$ imply that for each observable $A$, we have

$$
\mathscr{E}^{A}(\Delta)(B)=P^{A}(\Delta) \mathscr{E}^{A}(R)(B)
$$

for each $\Delta \in B(R)$ and $B \in B(\mathscr{H})$, where $\mathscr{E}^{A}(R)$ is a projection mapping of norm one of $B(\mathscr{H})$ onto the von Neumann algebra $\mathscr{U}_{A}^{\prime}$.

Proof. From the finite additivity property (B3), it follows that

$$
P^{A}(\Delta) \mathscr{E}^{A}(R)(B)=P^{A}(\Delta) \mathscr{E}^{A}(\Delta)(B)+P^{A}(\Delta) \mathscr{E}^{A}\left(\Delta^{\prime}\right)(B),
$$

for each $\Delta \in B(R)$, where $\Delta^{\prime}$ is the complement of $\Delta$ in $R$. All we must do now is to employ Eq. (4.8) for $\Delta$ and $\Delta^{\prime}$ in order to conclude that

$$
P^{A}(\Delta) \mathscr{E}^{A}(R) B=\mathscr{E}^{A}(\Delta) B,
$$

for all $B \in B(\mathscr{H})$.

In order to establish that $\mathscr{E}^{A}(R)$ is a norm one projection mapping onto $\mathscr{U}_{A}^{\prime}$, let us consider an arbitrary self-adjoint element $C \in \mathscr{U}_{A}^{\prime}$. Since $\mathscr{U}_{A}^{\prime}$ is a von Neumann algebra, we have $P^{C}(\Delta) \in \mathscr{U}_{A}^{\prime}$ for each $\Delta \in B(R)$. Now it follows from Lemma 4.2 that

$$
\mathscr{E}^{A}(R) P^{C}(\Delta)=P^{A}(R) P^{C}(\Delta)=P^{C}(\Delta),
$$

for each $\Delta \in B(R)$. Also, $\mathscr{E}^{A}(R)$ is continuous in the norm topology on $B(\mathscr{H})$, as it is a positive linear map. If we now recall the well-known fact that every bounded self-adjoint operator can be expressed as a limit under the norm topology of linear combinations of its spectral projectors, we can then conclude from Eq. (4.14) that

$$
\mathscr{E}^{A}(R) C=C
$$

for all self-adjoint elements $C \in \mathscr{U}_{A}^{\prime}$. It therefore follows that $\mathscr{U}^{A}(R)$ acts as the identity map on $\mathscr{U}_{A}^{\prime}$. Since we have already shown in Lemma 4.1 that the range of $\mathscr{U}^{A}(R)$ is contained in $\mathscr{U}_{A}^{\prime}$, we have the following

$$
\begin{aligned}
\text { Range } \mathscr{E}^{A}(R) & =\mathscr{U}_{A}^{\prime} \\
{\left[\mathscr{E}^{A}(R)\right]^{2} } & =\mathscr{E}^{A}(R) .
\end{aligned}
$$

In other words, $\mathscr{E}^{A}(R)$ is a projection map onto $\mathscr{U}_{A}^{\prime}$. Since we also have from $(B 2)$ that

$$
\mathscr{E}^{A}(R) I=I,
$$


it follows that $\mathscr{E} A(R)$ is a projection of norm one of $B(\mathscr{H})$ onto $\mathscr{U}_{A}^{\prime}$, thereby establishing the theorem.

Theorem 4.3 provides a more or less complete characterization of the expectations $\left\{\mathscr{E}^{A}(\Delta)\right\}$ specifying the "collapse" associated with an observable $A$, modulo the choice of the norm one projection mapping $\mathscr{E} A(R): B(\mathscr{H}) \rightarrow \mathscr{U}_{A}^{\prime}$, which will have to be chosen such that for observables with a purely discrete spectrum we recover the conventional von Neumann-Lüders collapse postulate (3.11). Before going into an explicit construction of such norm one projection maps, we shall first establish two very important conclusions which follow directly from the above theorem. The first one concerns the impossibility of formulating the collapse postulate in terms of operation-valued measures and may be stated as follows.

Theorem 4.4. If the assumptions $(A)-(D)$ are satisfied, then the expectations $\left\{\mathscr{E}^{A}(\Delta)\right\}$ associated with an observable $A$ cannot be normal unless $A$ has a purely discrete spectrum.

Proof. As we have shown in Theorem 4.3

$$
\mathscr{E}^{A}(\Delta)=P^{A}(\Delta) \mathscr{E}^{A}(R),
$$

where $\mathscr{E}^{A}(R)$ is a projection map of norm one of $B(\mathscr{H})$ onto $\mathscr{U}_{A}^{\prime}$. It is a classic result due to Tomiyama $[31,32]$ that a projection map of norm one of a von Neumann algebra onto a subalgebra satisfies all the properties characteristic of a so-called conditional expectation ${ }^{2}$ introduced earlier by Dixmier [33], Umegaki [34] and Nakamura and Turumuru [35]. If we now assume that $\mathscr{E}^{A}(R)$ is normal, then it follows that it is a normal conditional expectation of $B(\mathscr{H})$ onto the von Neumann algebra $\mathscr{U}_{A}^{\prime}$. However, it is well-known (see Davies [27]) that a normal condition expectation of $B(\mathscr{H})$ onto $\mathscr{U}_{A}^{\prime}=\left\{P^{A}(\Delta) \mid \Delta \in B(R)\right\}^{\prime}$ exists only if $A$ has a purely discrete spectrum. Hence $\mathscr{E}^{A}(\Delta)=P^{A}(\Delta) \mathscr{E}^{A}(R)$ cannot be normal unless $A$ has a purely discrete spectrum, thus establishing the above theorem.

The above theorem clearly demonstrates that we shall have to consider nonnormal expectations, in order to obtain a generalization of the conventional collapse postulate which is also consistent with the GBSF $(D)$. This of course rules out the possibility of employing operation-valued measures as they are the duals of normal expectation-valued measures as was noted earlier. In fact as non-normal expectations do not have a dual object (or adjoint) which is a transformation on $\mathscr{T}(\mathscr{H})$, we have arrived at a rather surprising result that the "collapse" associated with an observable with a continuous spectrum cannot be formulated as a transformation on the space of density operators. We shall return to this question later in Sect. V.

From a physical point of view, a somewhat more startling conclusion which follows from Theorem 4.3 (and hence from the GBSF) is that the joint probabilities involving observables with continuous spectra are not in general $\sigma$-additive. First of all it should be noted that the BSF implies that the probabilities $\operatorname{Pr}_{A}^{\rho}(\Delta)=\operatorname{Tr}\left(\rho P^{A}(\Delta)\right)$ associated with a single (instantaneous) observation are always $\sigma$-additive-whatever the observable $A$ might be. This of course is a

2 Some of these properties will be discussed in the next section. 
consequence of the fact that each $\rho \in \mathscr{T}(\mathscr{H})$ defines a "normal" or ultraweakly continuous linear functional on $B(\mathscr{H})$. However, the situation is very different when we consider joint probabilities, say $\operatorname{Pr}_{A, B}^{\rho}(R, \Delta)$ for $\Delta \in B(R)$. Let $\left\{\Delta_{i}\right\}$ be a countably infinite sequence of disjoint elements of $B(R)$ such that $\bigcup_{i} \Delta_{i}=\Delta$. We then have

$$
P^{B}(\Delta)=\sum_{i} P^{B}\left(\Delta_{i}\right),
$$

where the right-hand side converges in the strong and ultraweak topologies, but $n o t$ in the norm topology on $B(\mathscr{H})$. Now, the $\sigma$-additivity of the above joint probabilities is expressed by the equation

$$
\operatorname{Pr}_{A, B}^{\rho}(R, \Delta)=\sum_{i} \operatorname{Pr}_{A, B}^{\rho}\left(R, \Delta_{i}\right) .
$$

From Eqs. (3.17) and (4.2) we see that the above equation is equivalent to

$$
\operatorname{Tr}\left[\rho\left\{\mathscr{E}^{A}(R)\right\} P^{B}(\Delta)\right]=\sum_{i} \operatorname{Tr}\left[\rho\left\{\mathscr{E}^{A}(R)\right\} P^{B}\left(\Delta_{i}\right)\right],
$$

for all states $\rho$, which implies that

$$
\mathscr{E}^{A}(R) P^{B}(\Delta)=\sum_{i} \mathscr{E}^{A}(R) P^{B}\left(\Delta_{i}\right),
$$

where the right-hand side is required to converge in the ultraweak topology on $B(\mathscr{H})$. From Eqs. (4.18) and (4.21) it follows that the $\sigma$-additivity property (4.19) is satisfied for all observables $B$ only if $\mathscr{E}^{A}(R)$ is normal. This, as we have shown in Theorem 4.4, is possible only when $A$ has a purely discrete spectrum. In other words, we have established the following result.

Theorem 4.5. If assumptions $(A)-(D)$ are satisfied, then given any observable $A$ with a continuous spectrum, there exists a density operator $\rho$ and an observable $B$ such that the probabilities $\operatorname{Pr}_{A, B}^{\rho}(R, \Delta)$ are not $\sigma$-additive in $\Delta$.

We admit that the conclusion that we must deal with non- $\sigma$-additive probabilities is somewhat disturbing, for most of the traditional machinery of probability theory is built up on the basis of the assumption that the probabilities are $\sigma$-additive. However, as several probabilists have noted [36-38], $\sigma$-additivity is a purely technical requirement (but a very useful one at that) which has no basic empirical justification unlike the other assumptions such as the non-negativity and finite additivity of probabilities. Before going into a discussion of some of the intriguing features of a probability theory with only finitely additive probabilities (which shall be undertaken in Sect. VI), we shall first present an appropriate generalization of the conventional collapse postulate which is consistent with the assumptions $(A)-(D)$.

\section{The General Collapse Postulate}

In this section we shall introduce a generalization of the conventional collapse postulate which is more or less implied by the assumptions $(A)-(D)$ and the requirement that we should recover the conventional collapse postulate for observables 
with a purely discrete spectrum. As we saw in the previous section (cf. Theorem 4.3), the collapse associated with each observable $A$ is completely characterized by its spectral projectors $\left\{P^{A}(\Delta)\right\}$ and a norm one projection map $\mathscr{E} A(R): B(\mathscr{H}) \rightarrow \mathscr{U}_{A}^{\prime}$. We shall define a conditional expectation of $B(\mathscr{H})$ onto a von Neumann subalgebra $\mathscr{U}$ to be a linear self-adjoint map $\varepsilon: B(\mathscr{H}) \rightarrow \mathscr{U}$, which is also a norm one projection of $B(\mathscr{H})$ onto $\mathscr{U}$. It may be noted that the term conditional expectation is often used for several related (and sometimes even very different) notions in the literature $[9,10,27,30-35,39-50]$. Tomiyama $[31,32]$ has shown that a conditional expectation $\varepsilon: B(\mathscr{H}) \rightarrow \mathscr{U}$ has also the following properties

(i) $\varepsilon$ is positive,

(ii) $\varepsilon(A X B)=A \varepsilon(X) B$,

for all $A, B \in \mathscr{U}$ and $X \in B(\mathscr{H})$ (module property).

(iii) $\varepsilon\left(X^{*}\right) \varepsilon(X) \leqq \varepsilon\left(X^{*} X\right)$,

for all $X \in B(\mathscr{H})$.

It is also well known $[47,50]$ that a conditional expectation is a completely positive map. There have been several investigations [27, 32, 40-43] as to when there exists a normal conditional expectation onto a given subalgebra. As we stated in the previous section, it is well-known (see Davies [27]) that there exists a normal conditional expectation of $B(\mathscr{H})$ onto the von Neumann algebra $\mathscr{U}_{A}^{\prime}$ iff $A$ has a purely discrete spectrum.

In order to generalize the collapse postulate to arbitrary observables we need to consider the following questions:

(i) Does there always exist a conditional expectation $\varepsilon^{A}$ of $B(\mathscr{H})$ onto $\mathscr{U}_{A}^{\prime}$ for each observable $A$ ?

(ii) If there exist several such conditional expectations, can we fix a rule of association $A \rightarrow \varepsilon^{A}$, such that for an observable with a purely discrete spectrum $\varepsilon^{A}$ will be identical to the $\mathscr{E}^{A}(R)$ given by Eq. (3.11) (so that we can recover the conventional collapse postulate)?

We shall now see that the answers to both these questions are in the affirmative. In fact we shall explicitly construct a class of conditional expectations onto $\mathscr{U}_{A}^{\prime}$ following a procedure essentially due to Arvenson [41]. For this purpose we shall employ the other canonical object (apart from the spectral projectors) that is associated with each observable via Stone's theorem - the strongly continuous one-parameter group of unitary operators $\left\{e^{i t A} \mid t \in R\right\}$ generated by $A$. First of all let us note the following important result which is a consequence of Stone's theorem

$$
\mathscr{U}_{A}^{\prime} \equiv\left\{P^{A}(\Delta) \mid \Delta \in B(R)\right\}^{\prime}=\left\{e^{i t A} \mid t \in R\right\}^{\prime} .
$$

Eq. (5.3) implies that $\mathscr{U}_{A}^{\prime}$ is also the fixed point algebra for the group of automorphisms $B \rightarrow e^{i t A} B e^{-i t A}$ defined on $B(\mathscr{H})$. Since our construction of the conditional expectation depends on the notion of an invariant mean on the additive group $R$ of real numbers, we shall briefly summarize the main properties of invariant means on $R$ (see for example $[51,52]$ ).

Let $C_{B}(R)$ denote the Banach space of all bounded continuous complex- 
valued functions on $R$ with the norm

$$
\|f\|=\sup _{x \in R}|f(x)| \text {. }
$$

An invariant mean $\eta$ on the additive group $R$ is a positive linear functional on $C_{B}(R)$ such that the following properties are satisfied:

(i) If $e \in C_{B}(R)$ is the function $e(x)=1$ for all $x \in R$, then

$$
\eta(e)=1 \text {. }
$$

(ii) For each $a \in R$,

$$
\eta\left(f_{a}\right)=\eta(f)
$$

where $f_{a}$ is the function

$f_{a}(x)=f(x+a)$.

We shall employ the notation $\eta_{x} f(x)$ to indicate the number $\eta(f)$ whenever the function $f(x)$ is explicitly given. From the above definition, it is obvious that

$$
|\eta(f)| \leqq\|f\|
$$

for all $f \in C_{B}(R)$. It may also be noted that there are several invariant means on $R$. However, it is well known [52] that if $f \in C_{B}(R)$ is also a weakly almost periodic function, we then have

$$
\eta \underset{x}{\eta f}(x)=\lim _{T \rightarrow \infty} \frac{1}{2 T} \int_{-T}^{T} f(x) d x
$$

for every invariant mean $\eta$ on $R$.

The basic result concerning the existence of conditional expectations onto $\mathscr{U}_{A}^{\prime}$ may now be stated as follows.

Theorem 5.1. Let $\eta$ be an invariant mean on the additive group $R$. Then, for each observable A there exists a unique conditional expectation $\varepsilon_{\eta}^{A}$ of $B(\mathscr{H})$ onto $\mathscr{U}_{A}^{\prime}$ such that

$$
\operatorname{Tr}\left[v \varepsilon_{\eta}^{A}(B)\right]=\eta_{x} \operatorname{Tr}\left[v e^{i A x} B e^{-i A x}\right]
$$

for all $v \in \mathscr{T}(\mathscr{H})$ and $B \in B(\mathscr{H})$.

Proof. The function

$$
x \rightarrow \operatorname{Tr}\left[v e^{i A x} B e^{-i A x}\right]
$$

defined on the real line is continuous and bounded by $\|v\|_{1}\|B\|$ (where \|\|$_{1}$ denotes the trace norm on $\mathscr{T}(\mathscr{H})$ ). Hence, the map

$$
v \rightarrow \eta_{x} \operatorname{Tr}\left[v e^{i A x} B e^{-i A x}\right]
$$

defines a bounded linear functional on $\mathscr{T}(\mathscr{H})$ so that there exists a unique operator 
$\varepsilon_{\eta}^{A}(B) \in B(\mathscr{H})$ such that

$$
\operatorname{Tr}\left(v \varepsilon_{\eta}^{A}(B)\right)=\eta \operatorname{Tr}\left(v e^{i A x} B e^{-i A x}\right)
$$

for all $B \in B(\mathscr{H})$ and $v \in \mathscr{T}(\mathscr{H})$.

The linearity and positivity of the map $\varepsilon_{\eta}^{A}: B(\mathscr{H}) \rightarrow B(\mathscr{H})$, follow from the corresponding properties of the invariant mean $\eta$. The property

$$
\varepsilon_{\eta}^{A}(I)=I
$$

follows from Eq. (5.5). In order to show that $\varepsilon_{\eta}^{A} B \in \mathscr{U}_{A}^{\prime}$, we consider the following relation, which follows from (5.10) and (5.6)

$$
\begin{aligned}
\operatorname{Tr}\left(v e^{i x^{\prime} A} \varepsilon_{\eta}^{A}(B) e^{-i x^{\prime} A}\right) & =\prod_{x} \operatorname{Tr}\left(v e^{i\left(x+x^{\prime}\right) A} B e^{-i\left(x+x^{\prime}\right) A}\right. \\
& =\eta_{x} \operatorname{Tr}\left(v e^{i x A} B e^{-i x A}\right)=\operatorname{Tr}\left(v \varepsilon_{\eta}^{A}(B)\right),
\end{aligned}
$$

which holds for all $v \in \mathscr{T}(\mathscr{H})$ so that

$$
e^{i x^{\prime} A} \varepsilon_{\eta}^{A}(B) e^{-i x^{\prime} A}=\varepsilon_{\eta}^{A}(B)
$$

for all $x^{\prime} \in R$. We can therefore conclude, on the basis of Eq. (5.3), that $\varepsilon_{\eta}^{A}(B) \in \mathscr{U}_{A}^{\prime}$ for all $B \in B(\mathscr{H})$. Finally, if $B \in \mathscr{U}_{A}^{\prime}$, it is obvious from the relations (5.10) and (5.5) that $\varepsilon_{\eta}^{A}(B)=B$. Thus we have verified that $\varepsilon_{\eta}^{A}$ satisfies all the properties characteristic of a conditional expectation of $B(\mathscr{H})$ onto $\mathscr{U}_{A}^{\prime}$, thus establishing the above theorem.

As regards the second question that was posed above, we have the following rather interesting result.

Theorem 5.2. If $A$ is an observable with a purely discrete spectrum, and its spectral resolution is given by

$$
A=\sum_{i} \lambda_{i} P_{i}
$$

then for each $B \in B(\mathscr{H})$ we have

$$
\varepsilon_{\eta}^{A}(B)=\sum_{i} P_{i} B P_{i}
$$

for all invariant means $\eta$ on the real line.

Proof. If the observable $A$ is given by (5.11), we then have for each $B \in B(\mathscr{H})$ and $v \in \mathscr{T}(\mathscr{H})$

$$
\operatorname{Tr}\left(v e^{i x A} B e^{-i x A}\right)=\sum_{i, j} e^{i\left(\lambda_{\imath}-\lambda_{j}\right) x} \operatorname{Tr}\left(v P_{i} B P_{j}\right),
$$

which can easily be verified to be an almost periodic function on the real line (see for example [53] and references cited therein). We can now employ Eq. (5.9) and the fact that the right-hand side converges uniformly, to obtain

$$
\begin{aligned}
\eta_{x} \operatorname{Tr}\left(v e^{i x A} B e^{-i x A}\right) & =\sum_{i, j} \operatorname{Tr}\left(v P_{i} B P_{j}\right) \lim _{T \rightarrow \infty} \frac{1}{2 T} \int_{-T}^{T} e^{i\left(\lambda_{i}-\lambda_{j}\right) x} d x \\
& =\sum_{i} \operatorname{Tr}\left(v P_{i} B P_{i}\right) .
\end{aligned}
$$


Equation (5.10) and the ultraweak continuity of $v \in \mathscr{T}(\mathscr{H})$ when regarded as a functional on $B(\mathscr{H})$, yield the relation

$$
\varepsilon_{\eta}^{A}(B)=\sum_{i} P_{i} B P_{i},
$$

which establishes the above theorem.

From Theorems (5.1) and (5.2) it is clear that we can now set $\mathscr{E}^{A}(R)=\varepsilon_{\eta}^{A}$ for each observable $A$. In other words, we can now employ for the "collapse" associated with each observable $A$, the expectations

$$
\mathscr{E}^{A}(\Delta)=P^{A}(\Delta) \varepsilon_{\eta}^{A}
$$

If we now emply Eq. (5.12) we then obtain, as a particular case of Eq. (5.14), the conventional collapse postulate (cf. Eq. (3.11))

$$
\mathscr{E}^{A}(\Delta) B=P^{A}(\Delta) \sum_{i} P_{i} B P_{i}=\sum_{\lambda_{i} \in \Delta} P_{i} B P_{i},
$$

for every observable $A$ with a purely discrete spectrum as given by (5.11). It is also clear from our discussion that the collapse postulate as given by Eq. (5.14) is more or less an inevitable consequence of the assumptions $(A)-(D)$.

However, we are still left with the arbitrariness in the choice of the invariant mean $\eta$ employed in Eq. (5.14), and as we have noted, there do exist infinitely many invariant means on the additive group $R$. As we shall later see in a concrete example, different choices of the invariant mean do lead to different predictions for the joint probabilities. For the purposes of the present investigation, we shall leave the choice of the particular invariant mean employed in the collapse postulate to be rather arbitrary. We do however hope that further investigations will throw some light on (i) either the physical motivation behind making a particular choice, or on (ii) the physical significance of employing different invariant means to characterize possibly the various different ways of measuring the same observable. If we now tentatively assume that a particular invariant mean $\eta$ has been chosen, then we are led to the following generalization of the collapse postulate as a natural consequence of our assumptions $(A)-(D)$.

E. The general collapse postulate: For each observable A, the associated finitely additive expectation-valued measure $\Delta \rightarrow \mathscr{E}^{A}(\Delta)$ is given by

$$
\mathscr{E}^{A}(\Delta)=P^{A}(\Delta) \varepsilon^{A}
$$

for each $\Delta \in B(R)$, where the conditional expectation $\varepsilon^{A}: B(\mathscr{H}) \rightarrow \mathscr{U}_{A}^{\prime}$ is given by

$$
\operatorname{Tr}\left[v \varepsilon^{A}(B)\right]=\eta_{x} \operatorname{Tr}\left[v e^{i x A} B e^{-i x A}\right]
$$

for all $v \in \mathscr{T}(\mathscr{H})$ and $B \in B(\mathscr{H})$.

Having arrived at the general collapse postulate we can now adopt $(A),(C)$ and $(E)$ as the basic postulates of quantum theory. Of course the assumptions $(B)$ and $(D)$ are now automatically satisfied and we also have a non-trivial generalization of the conventional framework, wherein we can discuss the joint probabilities of any sequence of observations. For example, we can employ $(E)$ and write 
down explicitly the generalization (3.17) of the conventional Wigner formula (2.5), as follows:

$$
\begin{aligned}
\operatorname{Pr}_{A_{1}\left(t_{1}\right), A_{2}\left(t_{2}\right), \ldots, A_{r}\left(t_{r}\right)}^{\rho}\left(\Delta_{1}, \Delta_{2}, \ldots \Delta_{r}\right)=\eta \eta \ldots \eta\left[\operatorname { T r } \rho \left\{P^{A_{1}\left(t_{1}\right)}\left(\Delta_{1}\right)\right.\right. & \\
x_{1} x_{2} & x_{r}-1 \\
& \cdot e^{i x_{1} A_{1}\left(t_{1}\right)} P^{A_{2}\left(t_{2}\right)}\left(\Delta_{2}\right) e^{i x_{2} A_{2}\left(t_{2}\right)} \ldots e^{i x_{r-1} A_{r-1}\left(t_{r}-1\right)} P^{A_{r}\left(t_{r}\right)}\left(\Delta_{r}\right) \\
& \left.\cdot e^{-i x_{r-1} A_{r-1}\left(t_{r-1}\right)} \ldots e^{-i x_{2} A_{2}\left(t_{2}\right)} e^{-i x_{1} A_{1}\left(t_{1}\right)}\right]
\end{aligned}
$$

The generalized Wigner formula (5.18) is a non-trivial generalization of (2.5) and (2.6) as it is valid for arbitrary observables $\left\{A_{i}\left(t_{i}\right)\right\}$ and reduces to (2.5) and (2.6) for observables with a purely discrete spectrum. As an explicit application of (5.18), we can consider a one-dimensional non-relativistic particle which is subjected to a measurement of momentum $P$ immediately followed by a measurement of position $Q-a$ situation that cannot be discussed in the conventional framework. This example also serves to illustrate the fact that the joint probabilities (5.18) do in fact depend on the choice of the invariant mean employed. For this purpose let us consider the closed and invariant subspace $L \subset C_{B}(R)$, which consists of those functions $f$ for which the limits $f( \pm \infty)=\lim _{x \rightarrow \pm \infty} f(x)$ exist.

From the Hahn-Banach and the Markov-Kakutani theorems (see for example [54]) it can be established that for each $0 \leqq \lambda \leqq 1$, there exists an invariant mean $\eta_{\lambda}$ on $C_{B}(R)$ such that

$$
\eta_{\lambda} f=\lambda f(-\infty)+(1-\lambda) f(\infty)
$$

for all $f \in L$. If we now consider the function

$$
f(x)=\operatorname{Tr}\left[v e^{i P x} P^{Q}([-\infty, a]) e^{-i P x}\right]
$$

for some finite $a$. It then follows from the canonical commutation relations that $f \in L$, as in fact $f(-\infty)=1, f(+\infty)=0$. If we now employ the invariant mean in the Wigner formula (5.18), it then follows that

$$
\operatorname{Pr}_{P, Q}^{\rho}(R,(-\infty, a])=\lambda
$$

for all finite $a$. Similarly we can show that

$$
\operatorname{Pr}_{P, Q}^{\rho}(R,[a,+\infty))=1-\lambda
$$

and

$$
\operatorname{Pr}_{P, Q}^{\rho}(R,[a, b])=0
$$

for all finite $a, b$.

The most important feature of the above equations (5.20)-(5.22) is that they very clearly show that immediately after a measurement of the momentum, the position distribution is entirely concentrated at $\pm \infty$. This fact does seem to throw some new light on the meaning of the uncertainly relations from the point of view of measurement theory. It may also be noted that results very similar to Eqs. (5.20)(5.22) can be derived for the probabilities $\operatorname{Pr}_{Q, P}^{\rho}(R,$.$) .$

Equations (5.20)-(5.22) also demonstrate the fact that the joint probabilities (5.18) do depend on the particular invariant mean $\eta_{\lambda}$ employed. In this context it 
might be interesting to note that results some what similar to Eqs. (5.20)-(5.22) (particularly with $\lambda=1 / 2$ ) were obtained by Farrukh [19] on the basis of his approach through non-standard analysis. Farrukh was also led to a consideration of non- $\sigma$-additive joint probabilities for observables with continuous spectra. Though our results are obtained by entirely standard methods, we suspect that there is perhaps some deep connection between the approach of Farrukh and the one outlined here, a study of which might also clarify the question of the choice of the invariant mean in Eq. (5.18). Another possibility for resolving this question could be via a study of the limiting process envisaged by von Neumann (see the discussion in Sect. II), either directly or perhaps in terms of the approximate position and momentum measurements introduced by Davies [10,27].

We shall now collect, in the following theorem, some of the important properties of the collapse associated with an observable as given by the general collapse postulate $(E)$.

Theorem 5.3. If $A, B$ are any two observables, then we have the following:

i) $\mathscr{E}^{A}\left(\Delta_{1}\right) \mathscr{E}^{A}\left(\Delta_{2}\right)=\mathscr{E}^{A}\left(\Delta_{1} \cap \Delta_{2}\right)$

for all $\Delta_{1}, \Delta_{2} \in B(R)$.

ii) If $A$ and $B$ are compatible, then

$$
\mathscr{E}^{A}\left(\Delta_{1}\right) \mathscr{E}^{B}\left(\Delta_{2}\right)=\mathscr{E}^{B}\left(\Delta_{2}\right) \mathscr{E}^{A}\left(\Delta_{1}\right)
$$

for all $\Delta_{1}, \Delta_{2} \in B(R)$.

iii) $\varepsilon^{A}=\varepsilon^{B} \Leftrightarrow \mathscr{U}_{A}^{\prime}=\mathscr{U}_{B}^{\prime}$

iv) If $f: R \rightarrow R$ is a Borel function which also induces a Borel isomorphism of the spectrum of $A$ onto some Borel set in $R$, then

$$
\varepsilon^{f(A)}=\varepsilon^{A}
$$

and

$$
\mathscr{E}^{f(A)}(\Delta)=\mathscr{E}^{A}\left(f^{-1}(\Delta)\right)
$$

for all $\Delta \in B(R)$.

Proof. The property i) follows directly from the relation

$$
\mathscr{E}^{A}(\Delta)=P^{A}(\Delta) \varepsilon^{A},
$$

once we employ the module property (5.1) of the conditional expectation $\varepsilon^{A}$. In order to prove ii), we shall first show that if $A, B$ are compatible, we then have

$$
\varepsilon^{A} \varepsilon^{B}=\varepsilon^{B} \varepsilon^{A}
$$

For this let us consider an arbitrary element $C \in B(\mathscr{H})$. Since $A, B$ are compatible, we have that for each $v \in \mathscr{T}(\mathscr{H})$

$$
\begin{aligned}
\operatorname{Tr} & {\left[v \varepsilon^{A} \varepsilon^{B}(C)\right] } \\
& =\eta \eta \operatorname{Tr}\left[v e^{i x A} e^{i x^{\prime} B} C e^{-i x^{\prime} B} e^{-i x A}\right] \\
& x x^{\prime} \\
= & \eta \eta \operatorname{Tr}\left[v e^{i x^{\prime} B} e^{i x A} C e^{-i x A} e^{-i x^{\prime} B}\right] \\
& x x^{\prime} \\
& =\eta_{x} \operatorname{Tr}\left[v \varepsilon^{B}\left(e^{i x A} C e^{-i x A}\right)\right]
\end{aligned}
$$


But since $e^{i x A} \in \mathscr{U}_{B}^{\prime}$, we have from the module property (5.1) the relation

$$
\varepsilon^{B}\left(e^{i x A} C e^{-i x A}\right)=e^{i x A} \varepsilon^{B}(C) e^{-i x A}
$$

From (5.29) and (5.30) we can conclude that

$$
\operatorname{Tr}\left[v \varepsilon^{A} \varepsilon^{B} C\right]=\operatorname{Tr}\left[v \varepsilon^{B} \varepsilon^{A} C\right]
$$

for all $v \in \mathscr{T}(\mathscr{H})$, from which it follows that

$$
\varepsilon^{A} \varepsilon^{B} C=\varepsilon^{B} \varepsilon^{A} C
$$

for all $C \in B(\mathscr{H})$, which implies Eq. (5.28). The property ii) can now easily be derived from (5.28) by apply Eq. (5.26) and the module property (5.1).

As regards iii), the implication $\varepsilon^{A}=\varepsilon^{B} \Rightarrow \mathscr{U}_{A}^{\prime}=\mathscr{U}_{B}^{\prime}$ is trivial as the algebras $\mathscr{U}_{A}^{\prime}, \mathscr{U}_{B}^{\prime}$ are the ranges of $\varepsilon^{A}, \varepsilon^{B}$, respectively. To prove the converse, we may first note that if $\mathscr{U}_{A}^{\prime}=\mathscr{U}_{B}^{\prime}$, then $A, B$ are compatible, so that we have $\varepsilon^{A} \varepsilon^{B}=\varepsilon^{B} \varepsilon^{A}$ as shown above. Also, since our hypothesis $\mathscr{U}_{A}^{\prime}=\mathscr{U}_{B}^{\prime}$ implies that $\varepsilon^{A} C \in \mathscr{U}_{B}^{\prime}$, $\varepsilon^{B} C \in \mathscr{U}_{A}^{\prime}$ for all $C \in B(\mathscr{H})$, we get

$$
\varepsilon^{A} C=\varepsilon^{B} \varepsilon^{A} C=\varepsilon^{A} \varepsilon^{B} C=\varepsilon^{B} C
$$

for all $C \in B(\mathscr{H})$, so that we have $\varepsilon^{A}=\varepsilon^{B}$. Finally, in order to prove iv), we note that if the function $f$ satisfies the above conditions, then every spectral projector of $A$ is also a spectral projector of $f(A)$ and vice versa. Hence we have $\mathscr{U}_{A}^{\prime}=\mathscr{U}_{f(A)}^{\prime}$, so that we can conclude from iii) that $\varepsilon^{A}=\varepsilon^{f(A)}$. Equation (5.27) follows right away from (5.26) once we make use of the relation (2.9) between the spectral projectors of $A$ and $f(A)$. This completes the proof of the above theorem.

We shall comment briefly on the physical significance of the various properties established in the above theorem. The property (i) is sometimes referred to as the repeatability property of the collapse transformation. It may be noted in this connection that Davies and Lewis [9] had suggested that it might perhaps be necessary to give up this property in order to obtain an appropriate generalization of the collapse postulate. Property (ii) shows that for compatible observables, the associated collapse transformations also commute. (iii) shows that the conditional expectation $\varepsilon^{A}$ is completely determined by just the algebra $\mathscr{U}_{A}^{\prime}$ associated with $A$. Finally, (iv) is just a generalization to an arbitrary observable of the result stated in Lemma 2.1 for an observable with a purely discrete spectrum. We may note that it essentially demonstrates that the collapse associated with an observable $A$ and that associated with its function $f(A)$ are related only when $\mathscr{U}_{A}^{\prime}=\mathscr{U}_{f(A)}^{\prime}$.

Finally, a few remarks may be made on the novel feature of the collapse postulate $(E)$ that it cannot be formulated in terms of collapse transformations (or operations) which are defined on the space of density operators. As was noted in Sect. IV, this is due to the fact that the expectations $\left\{\mathscr{E}^{A}(\Delta)\right\}$ as given by $(5.16)$, (5.17) are in general non-normal. In our opinion, this novel feature of the collapse postulate $(E)$ clarifies to some extent the essential role played by the collapse postulate - for it has often been thought of as a postulate which introduces some strange transformation in the state of a system "caused" by the act of observation. The necessary delinking of the collapse postulate from transformations defined on 
the set of states (which is implied in our postulate $(E)$ ), would perhaps serve to illustrate more clearly the fact that the main function of this postulate is to provide a prescription for the joint probabilities associated with the outcomes of successive observations.

It should however be noted that it is still possible to consider the postulate $(E)$ as introducing a transformation on the "states", provided we are willing to include as states all the normalized positive linear functionals on $B(\mathscr{H})$. It is well known that there exists an isometric isomorphism of $\mathscr{T}(\mathscr{H})$ into a subspace of the dual $B(\mathscr{H})^{*}$ of $B(\mathscr{H})$. Also, every expectation $\mathscr{E}^{A}(\Delta)$ (normal or otherwise) has an adjoint which is a positive linear map on $B(\mathscr{H})^{*}$. Hence, if we accept all the normalized positive elements of $B(\mathscr{H})^{*}$ as possible states of a system, then the collapse postulate $(E)$ can also be viewed as introducing certain collapse transformations on such states. This formulation would also vindicate the often expressed conjecture that a generalization of the collapse transformation (2.3) would, in the case of observables with continuous spectra, take every density operator into a more general object - which in our case would be an element of $B(\mathscr{H})^{*}$ which is not a normal linear functional on $B(\mathscr{H})$. The fact that the joint probabilities for observables turn out to be non- $\sigma$-additive will now also be closely related to the inclusion of non-normal linear functionals on $B(\mathscr{H})$ as possible states of a system. Finally, we may note that all our results can be easily extended to the algebraic formulations of quantum theory, wherein the significance of some of the above remarks would be more transparent.

\section{The Statistics of Successive Observations in Quantum Theory}

In this section we shall consider some of the important features of the framework developed so far, from the standpoint of probability theory. We have seen that the general collapse postulate $(E)$ provides an unambiguous and complete prescription for realizing various outcomes in any arbitrary sequence of observations, in terms of the generalized Wigner formula (5.18). It was also noted that these joint probabilities are not in general $\sigma$-additive. Before going into a discussion of the implications of non- $\sigma$-additivity on the statistics of successive observations, we shall first consider some of the other non-classical features of the joint probabilities themselves.

To start with, it may be noted that the basic joint probability prescription of the classical probability theory can also be formulated in a manner analogous to the generalized Wigner formula ((3.17) and (5.18)). For this purpose consider a classical probability space $(\Omega, B(\Omega))$, where $\Omega$ is a complete separable metric space and $B(\Omega)$ is the associated $\sigma$ algebra of Borel sets. Each observable is a real valued random variable $A: \Omega \rightarrow R$ and the characteristic functions $\left\{\chi_{A^{-1}(\Delta)}\right\}$ are the classical analogues of spectral projectors. Again if $M(\Omega)$ is the Banach space of all bounded complex measures on $B(\Omega)$ and $\mathscr{L}(\Omega)$ is the Banach space of all bounded complex measurable functions on $(\Omega, B(\Omega))$, then we have the bilinear form

$$
\langle\mu, X\rangle=\int X d \mu
$$


for all $\mu \in M(\Omega)$ and $X \in \mathscr{L}(\Omega)$. The important feature of classical probability theory is that $\mathscr{L}(\Omega)$ is an abelian algebra, and therefore the algebra of all those bounded observables compatible with any given observable will be the whole of $\mathscr{L}(\Omega)$ itself. Thus each of the conditional expectations $\varepsilon^{A}$ will reduce to the identity map on $\mathscr{L}(\Omega){ }^{3}$ Hence we have for each observable $A$, the associated expectations $\left\{\mathscr{E}^{A}(\Delta)\right\}$ given by

$$
\mathscr{E}^{\mathscr{A}}(\Delta) X=\chi_{A^{-1}(\Delta)} \varepsilon^{A} X=\chi_{A^{-1}(\Delta)} X
$$

for each $X \in \mathscr{L}(\Omega)$. The generalized Wigner formula (3.17) will now reduce to the following:

$$
\begin{aligned}
\operatorname{Pr}_{A_{1}, A_{2}, \ldots, A_{r}}^{\rho}\left(\Delta_{1}, \Delta_{2}, \ldots, \Delta_{r}\right) & =\left\langle\mu, \mathscr{E}^{A_{1}}\left(\Delta_{1}\right) \mathscr{E}^{A_{2}}\left(\Delta_{2}\right) \ldots \mathscr{E}^{A A_{r}}\left(\Delta_{r}\right) I\right\rangle \\
& =\int \chi_{A_{1}^{-1}\left(\Delta_{1}\right)} \chi_{A_{2}^{-1}\left(\Delta_{2}\right)} \ldots \chi_{A_{r}^{-1}\left(\Delta_{r}\right)} d \mu \\
& =\mu\left(A_{1}^{-1}\left(\Delta_{1}\right) \cap A_{2}^{-1}\left(\Delta_{2}\right) \cap \ldots \cap A_{r}^{-1}\left(\Delta_{r}\right)\right)
\end{aligned}
$$

which is the well-known prescription for joint probabilities in classical probability theory.

It is thus clear that the non-classical features of quantum-theoretic joint probabilities arise from the fact that $B(\mathscr{H})$ is a non-abelian algebra so that the conditional expectations $\varepsilon^{A}: B(\mathscr{H}) \rightarrow \mathscr{U}_{A}^{\prime}$ turn out to be nontrivial. It is precisely this fact that $\varepsilon^{A}$ is not the identity map (whatever the observable $A$ might be) which gives rise to the "quantum interference of probabilities" [24-26] - which essentially is the feature of quantum-theoretic joint (and conditional) probabilities that they crucially depend also on the entire sequence of observations performed on a system.

Apart from the quantum interference of probabilities which was also well known earlier in connection with observables with a purely discrete spectrum, the important non-classical feature of the quantum-theoretic joint probabilities (which arises only when we include in our considerations observables with continuous spectra) is that they are not $\sigma$-additive in general. For example, as we already noted, the joint probability $\operatorname{Pr}_{A, B}^{\rho}\left(\Delta_{1}, \Delta_{2}\right)$ is not $\sigma$-additive in $\Delta_{2}$ whenever $A$ has a continuous spectrum, because the conditional expectation $\varepsilon^{2}$ turns out to be non-normal. If in addition the observable $A$ happens to have a purely continuous and simple spectrum, then we can show that the associated conditional expectation will be "singular" $[32,43]$ and has the property

$$
\varepsilon^{A} C=0
$$

for each compact operator $C$. We therefore have the following rather curious

3 It should of course be emphasised that non-trivial conditional expectations do arise in classical probability theory when we consider, say, the algebra $\mathscr{U}_{A}$ of all bounded complex-valued Borel functions of $A$ [55]. In fact the study of conditional expectations in operator algebras was inspired mainly by the work on conditional expectations in classical probability theory. However, unlike in quantum theory, the conditional expectations play no role in the formulation of the basic prescriptions of classical probability theory 
result:

$$
\operatorname{Pr}_{A, B}^{\rho}(\Delta,\{\lambda\})=0,
$$

for all $\Delta \in B(R)$, whenever $A$ has a purely continuous simple spectrum and $\lambda$ is an eigenvalue of $B$ with a finite multiplicity.

The most important limitation of a probability theory with only finitely additive probabilities seems to be that we no longer have the very well developed machinery of integration with respect to a $\sigma$-additive probability measure which plays a very crucial role in the classical probability theory in providing a natural definition of expectation values, correlation functions etc. In fact the joint probabilities $\operatorname{Pr}_{A_{1}\left(t_{1}\right), A_{2}\left(t_{2}\right), \ldots, A_{r}\left(t_{r}\right)}^{\rho}\left(\Delta_{1}, \Delta_{2}, \ldots, \Delta_{r}\right)$ given by the generalized Wigner formula (5.18) are just finitely additive set functions, which are in addition defined only on finite Unions of sets of the form $\Delta_{1} \times \Delta_{2} \times \ldots \times \Delta_{r}$, where $\Delta_{i} \in B(R)$ for each $i \in\{1,2, \ldots, r\}$. Hence, there does not seem to be any immediate prescription ${ }^{4}$ for the evaluation of the expectation value of an arbitrary Borel function $f: R^{r} \rightarrow R$, based solely on the generalized Wigner formula (5.18). From a physical point of view, this is indeed a very serious limitation, as one usually employs the probabilities only insofar as they determine the entire statistics of successive observations - which essentially involves a determination of the mean or expectation values of all suitable functions of the set of outcomes when an arbitrary sequence of observations are performed. For the purposes of the present investigation, we shall take the viewpoint that in order to have a complete theory, the expectation values of the various observations and their Borel functions are to be prescribed separately, but in a manner consistent with the basic prescription for probabilities. We shall now introduce such a prescription for expectation values, which (together with our assumptions $(A)$. $(C)$ and $(E))$ serves to characterize completely the statistics of any sequence of observations.

First of all it may be noted that for each state $\rho$ and arbitrary observables $\left\{A_{1}, A_{2}, \ldots, A_{r}\right\}$ the map

$$
\begin{aligned}
& \Delta_{1} \times \Delta_{2} \times \ldots \times \Delta_{r} \rightarrow \operatorname{Tr}\left[\rho P^{A_{1}}\left(\Delta_{1}\right) e^{i x_{1} A_{1}} \ldots e^{i x_{r-1} A_{r-1}} P^{A_{r}}\left(\Delta_{r}\right) e^{-i x_{r}-1 A_{r-1}}\right. \\
& \left.\ldots e^{-i x_{2} A_{2}} e^{-i x_{1} A_{1}}\right]
\end{aligned}
$$

can be extended into a unique (bounded and $\sigma$-additive) complex measure on $R^{r}$. The associated distribution function is given by

$$
\begin{aligned}
F_{A_{1}, A_{2}, \ldots, A_{r}}^{\rho}\left(\lambda_{1}, \lambda_{2}, \ldots, \lambda_{r} ; x_{1}, x_{2}, \ldots, x_{r-1}\right)= & \operatorname{Tr}\left[\rho P^{A_{1}}\left(\left(-\infty, \lambda_{1}\right]\right) e^{i x_{1} A_{1}} \ldots\right. \\
& \cdot e^{i x_{r}-1 A_{r}-1} P^{A_{r}}\left(\left(-\infty, \lambda_{r}\right]\right) e^{-i x_{r-1} A_{r-1}} \\
& \left.\ldots e^{-i x_{2} A_{2}} e^{-i x_{1} A_{1}}\right] .
\end{aligned}
$$

If $f: R^{r} \rightarrow R$ is a measurable function and $t_{1}<t_{2}<\ldots<t_{r}$, then we denote by $\operatorname{Exp}_{A_{1}\left(t_{1}\right), \ldots, A_{r}\left(t_{r}\right)}^{\rho}\left[f\left(A_{1}\left(t_{1}\right), \ldots, A_{r}\left(t_{r}\right)\right)\right]$, the expectation value of the function $f$ of the outcomes which are obtained when an ensemble of systems prepared in state $\rho$

4 Since the joint probability $\operatorname{Pr}_{A_{1}\left(t_{1}\right), \ldots, A_{r}\left(t_{r}\right)}^{\rho}\left(\Delta_{1}, \Delta_{2}, \ldots, \Delta_{r}\right)$ are not defined on the entire $\sigma$-algebra $B\left(R^{r}\right)$, we cannot, for example, employ the integration theory for finitely additive measures outlined in $[56, \mathrm{p} .354]$. 
are subjected to a sequence of experiments to observe $\left\{A_{1}\left(t_{1}\right), A_{2}\left(t_{2}\right), \ldots, A_{r}\left(t_{r}\right)\right\}$. We now introduce the following prescription for such expectation values.

(F) Expectation value postulate: Let $f: R^{r} \rightarrow R$ be a measurable function and $t_{1}<t_{2}<\ldots<t_{r}$. Then $\operatorname{Exp}_{A_{1}\left(t_{1}\right), \ldots, A_{r}\left(t_{r}\right)}^{\rho}\left[f\left(A_{1}\left(t_{1}\right), \ldots, A_{r}\left(t_{r}\right)\right]\right.$ exists and is given by

$$
\begin{aligned}
& \operatorname{Exp}_{A_{1}\left(t_{1}\right), A_{2}\left(t_{2}\right), \ldots, A_{r}\left(t_{r}\right)}^{\rho}\left[f\left(A_{1}\left(t_{1}\right), \ldots, A_{r}\left(t_{r}\right)\right)\right] \\
& =\eta \eta \eta \ldots \ldots \eta \quad\left[\int f\left(\lambda_{1}, \lambda_{2}, \ldots, \lambda_{r}\right)\right. \\
& \quad x_{1} x_{2} x_{r-1} \\
& \left.\quad \cdot d F_{A\left(t_{1}\right), \ldots, A_{r}\left(t_{r}\right)}^{\rho}\left(\lambda_{1}, \lambda_{2}, \ldots, \lambda_{r} ; x_{1}, x_{2}, \ldots, x_{r-1}\right)\right],
\end{aligned}
$$

whenever the right-hand side of the above equation exists.

The condition that the right-hand side of Eq. (6.7) exist is essentially the condition that the Lebesgue-Stieltjes integral enclosed by brackets [ ] which function (in all the variables $x_{1}, x_{2}, \ldots, x_{r-1}$ ) should be such that the operation of taking invariant means makes sense.

The expectation values defined by postulate $(F)$ satisfy the following properties, which also show its consistency with the collapse postulate $(E)$ and the predictions of conventional quantum theory (wherever available).

i) If $f_{1}, f_{2}$ are Borel functions on $R^{r}$, and $\alpha_{1}, \alpha_{2}$ are arbitrary real numbers, then

$$
\begin{aligned}
\operatorname{Exp}_{A_{1}\left(t_{1}\right), \ldots, A_{r}\left(t_{r}\right)}^{\rho}\left(\alpha_{1} f_{1}+\alpha_{2} f_{2}\right)= & \alpha_{1} \operatorname{Exp}_{A_{1}\left(t_{1}\right), \ldots, A_{r}\left(t_{r}\right)}^{\rho}\left(f_{1}\right) \\
& +\alpha_{2} \operatorname{Exp}_{A_{1}\left(t_{1}\right), \ldots, A_{r}\left(t_{r}\right)}^{\rho}\left(f_{2}\right),
\end{aligned}
$$

which shows the linearity of expectations.

ii) Expectation value of a constant function is constant.

iii) If $f: R^{r} \rightarrow R$ is given by

$$
f\left(\lambda_{1}, \lambda_{2}, \ldots, \lambda_{r}\right)=\chi_{\Delta_{1}}\left(\lambda_{1}\right) \chi_{\Delta_{2}}\left(\lambda_{2}\right) \ldots \chi_{\Delta_{r}}\left(\lambda_{r}\right)
$$

where $\Delta_{i} \in B(R), i=1,2, \ldots, r$, then

$$
\operatorname{Exp}_{A_{1}\left(t_{1}\right), \ldots, A_{r}\left(t_{r}\right)}^{\rho}(f)=\operatorname{Pr}_{A_{1}\left(t_{1}\right), \ldots, A_{r}\left(t_{r}\right)}^{\rho}\left(\Delta_{1}, \Delta_{2}, \ldots, \Delta_{r}\right),
$$

which shows the consistency of $(F)$ with $(E)$.

iv) If $\left\{A_{1}, A_{2}, \ldots, A_{r}\right\}$ is a compatible set of observables and $f: R^{r} \rightarrow R$ is a Borel function, then

$$
\operatorname{Exp}_{A_{1}, A_{2}, \ldots, A_{r}}^{\rho}(f)=\operatorname{Tr}\left(\rho f\left(A_{1}, A_{2}, \ldots, A_{r}\right)\right)
$$

whenever the right-hand side exists, which shows the consistency of $(F)$ with the predictions of conventional quantum theory. In particular, we recover the standard result

$$
\operatorname{Exp}_{A}^{\rho}(A)=\operatorname{Tr}(\rho A)
$$

whenever the right-hand side exists.

We shall not go into a further discussion of the statistics of successive observations, as would follow from the postulate $(F)$ except to note that the postulate $(F)$ reduces to the usual prescription for the expectation values in classical probability theory, if we assume that the algebra of observables is abelian. It may 
be of some interest also to write down the following formula, which we believe would be of importance in applications,

$$
\operatorname{Exp}_{A_{1}, A_{2}, \ldots, A_{r}}^{\rho}\left(A_{1}^{m_{1}} A_{2}^{m_{2}} \ldots A_{r}^{m_{r}}\right)=\operatorname{Tr}\left[\rho A_{1}^{m_{1}} \varepsilon^{A_{1}}\left(A_{2}^{m_{2}} \varepsilon^{A_{2}}\left(\ldots \varepsilon^{A_{r-1}}\left(A_{r}^{m_{r}}\right) \ldots\right)\right]\right.
$$

for all integers $m_{1}, m_{2}, \ldots, m_{r}$.

In conclusion we would like to emphasise that the postulates $(A),(C),(E)$ and $(F)$ provide a complete framework for the discussion of successive observations involving any arbitrary sequence of observables in quantum theory. The main observational prescriptions of the theory are contained in Eqs. (5.18) and (6.7), the former being a particular case of the latter if we take into account Eqs. (6.9) and (6.10). These of course include as particular cases all the basic observational prescriptions of conventional quantum theory. In addition, as we have shown, the general framework that has been introduced enables us to discuss situations (involving successive observables with a continuous spectrum) which cannot be handled in the conventional framework of quantum theory. It is hoped that this generalization would also perhaps give rise to new ways of confronting the elusive collapse postulate to appropriate experimental tests.

Acknowledgements. The author is much indebted to Dr. E. B. Davies for correcting an error in an earlier version and for several helpful suggestions. He is also grateful to Dr. S. Twareque Ali for useful correspondence on this subject. He would like to thank Professor Abdus Salam, the International Atomic Energy Agency and UNESCO for hospitality at the International Centre for Theoretical Physics, Trieste, where the final version of the manuscript was prepared.

\section{References}

1. Neumann, J. von: Mathematical foundations of quantum mechanics Princeton: Princeton University Press 1955

2. Lüders, G. : Ann. Phys. (Leipzig) (6) 8, 322 (1951)

3. Goldberger, M. L., Watson, K. : Phys. Rev. 134B, 919 (1964)

4. Furry, W. H. : Lectures in theoretical physics, Vol. VIIIA, (ed. W. E. Brittin). Boulder: University of Colorado Press 1966

5. Herbut, F. : Ann. Phys. (N.Y.) 55, 271 (1969); Int. J. Theoret. Phys. 11, 193 (1974)

6. Jammer, M. : The philosophy of quantum mechanics. New York: Wiley, 1974

7. d'Espagnat, B. : Conceptual foundations of quantum mechanics. New York: Benjamin, 1976

8. Gnanapragasam, B., Srinivas, M. D.: Pramana 12, 699, 1979

9. Davies, E. B., Lewis, J. T. : Commun. Math. Phys. 17, 293 (1969)

10. Davies, E. B. : J. Funct. Anal. 6, 318 (1970)

11. Prugovecki, E. : Quantum mechanics in hilbert space. New York: Academic Press 1971

12. Kristensen, P., Mejlbo, L., Poulsen, E. Y. : Commun. Math. Phys. 1, 175 (1964); Math. Scand. 14, 129 (1964); Commun. Math. Phys. 6, 29 (1967)

13. Grossman, A. : J. Math. Phys. 5, 1025 (1964); ibid. 6, 54 (1965); Commun. Math. Phys. 2, 1 (1966); ibid, 4, 203 (1967)

14. Böhm, in Lectures in theoretical physics, Vol. IXA, (eds. W. E. Brittin, A. O. Barut, M. Guenin). Yew York: Gordon and Breach, 1966

15. Roberts, J. E. : J. Math. Phys. 7, 1097 (1966); Commun. Math. Phys. 3, 98 (1966)

16. Antoine, J. P. : Math. Phys. 10, 53 (1969); ibid. 10, 2776 (1969)

17. Melsheimer, O. : J. Math. Phys. 15, 902 (1974); ibid. (1974)

18. Bogolubov, N. N., Logunov, A. A., Todorov, I. T. : Introduction to axiomatic quantum field theory. New York: Benjamin, 1975

19. Farrukh, M. O. : J. Math. Phys. 16, 177 (1975) 
20. Wigner, E. P. : Am. J. Phys. 31, 6 (1963)

21. Houtappel, R. M. F., van Dam, H., Wigner, E. P. : Rev. Mod. Phys. 37, 595 (1965)

22. Wigner, E. P. : Foundations of quantum mechanics. (ed. B. d'Espagnat) New York: Academic Press 1971

23. Wigner, E. P. : in: Contemporary research in the foundations and philosophy of quantum mechanics. (ed. C. A. Hooker.) Dordrecht: Reidel 1973

24. de Broglie, L. : Rev. Scientifique 259 (1948)

25. Srinivas, M. D.: J. Math. Phys. 16, 1672 (1975). Reprinted in: The Logico-algebraic approach to quantum theory, Vol. II, pp. 227-260, (ed. C. A. Hooker). Dordrecht: Reidel 1979

26. Srinivas, M. D.: J. Math. Phys. 19, 1705 (1978)

27. Davies, E. B.: Quantum theory of open systems. New York: Academic Press 1976

28. Schwinger, J. : Proc. Nat. Acad. Sci. (USA) 45, 1542 (1959); ibid. 46, 257 (1960); ibid. 46, 570 (1960)

29. Haag, R., Kastler, D. : J. Math. Phys. 5, 848 (1964)

30. Twareque Ali, S., Emch, G. G.: Covariant conditional expectations in quantum probability theory. ICTP, Trieste, preprint IC/74/72

31. Tomiyama, J. : Proc. Japan Acad. 33, 608 (1957)

32. Tomiyama, J. : Tensor products and projections of norm one in von Neumann algebras. Lecture notes, University of Copenhagen 1970

33. Dixmier, J. : Bull. Soc. Math. France 81, 9 (1953)

34. Umegaki, H. : Tohoku Math. J. 6, 177 (1954); ibid. 8, 86 (1956)

35. Nakamura, M., Turumuru, T. : Tohoku Math. J. 6, 182 (1954)

36. de Finetti, B. : Rend. Ist. Lomb. 63, 414 (1930); Ann. Triestini 19, 29 (1949); ibid. 20, (1950)

37. Kolmogorov, A. N. : Foundations of the theory of probability. New York: Chelsea 1950

38. Fine, T. : Theories of probability. New York: Academic Press 1973

39. Nakamura, M., Umegaki, H. : Math. Japon. 7, 151 (1962)

40. Kovacs, I., Szücs, J. : Acta Sci. Math. 27, 233 (1966)

41. Arveson, W. B. : Am. J. Math. 89, 572 (1967)

42. Takesaki, M. : J. Funct. Anal. 9, 306 (1972)

43. Tomiyama, J. : J. Funct. Anal. 10, 373 (1972)

44. Gudder, S. P., Marchand, J. P. : J. Math. Phys. 13, 799 (1972)

45. Guichardet, A. : Asterisque Nos. 13, 14, Soc. Math. de France, Paris 1974

46. Jajte, R. : Ann, Sci. Univ. Clermont 58, 11 (1975)

47. Stormer, E. : In: Foundations of quantum mechanics and ordered linear spaces. (eds. A. Hartkamper, H. Neumann) Berlin, Heidelberg, New York: Springer 1976

48. Maczynski, M. J. : Int. J. Theor. Phys. 16, 437 (1977)

49. Gudder, S. P., Marchand, J. P. : Rep. Math. Phys. 12, 317 (1977)

50. Evans, D. E., Lewis, J. T. : Commun. Dublin Inst. Adv. Studies A24, (1977)

51. Hewitt, E., Ross, K. A.: Abstract harmonic analysis. Vol. I. Berlin, Göttingen, Heidelberg: Springer 1963

52. Greenleaf, F. P. : Invariant means on topological groups. van Nostrand, Princeton 1969

53. Percival, I. C. : J. Math. Phys. 2, 235 (1961)

54. Dunford, N., Schwartz, J. : Linear operators Part I Inter Science, New York 1958

55. Moy, S. T. C. : Pac. J. Math. 4, 47 (1954)

56. Hewitt, E., Stromberg, K.: Real and abstract analysis. Berlin, Heidelberg, New York: Springer 1969

Communicated by R. Haag

Received May 10, 1979 